\title{
Hydrophobicity and Dynamic Characteristics of Cross-Linked Polystyrene Gels Studied with Fluorescent Probes
}

\author{
Kazuyuki Horie, ${ }^{*}$ Itaru Mita, ${ }^{*}$ Juheiji KaWABATA, ${ }^{* *}$ \\ Seiichi NAKAHAMA, ${ }^{* *}$ Akira HiraO, ${ }^{* *}$ \\ and Noboru YAMAZAKI** \\ *Institute of Space and Aeronautical Sciences, University of Tokyo, \\ Komaba, Meguro-ku, Tokyo 153, Japan. \\ **Department of Polymer Science, Tokyo Institute of Technology, \\ Ookayama, Meguro-ku, Tokyo 152, Japan.
}

(Received December 7, 1979)

\begin{abstract}
The hydrophobicity and dynamic characteristics of cross-linked polystyrene gels with quaternary ammonium groups (PSQA) have been studied from the fluorescence spectra and depolarization of 6-p-toluidino-2-naphthalenesulfonate (TNS) and 5-dimethylamino-1naphthalenesulfonate (DNS) in an aqueous dispersion of the ground PSQA samples. The positions of fluorescence maxima $\left(\lambda_{\mathrm{fmax}}\right)$ for both TNS and DNS are markedly shifted to the shorter wavelengths with increasing divinylbenzene content $(x)$ in PSQA. This shows an increase in the hydrophobicity of microenvironment around cationic sites in PSQA. The blue shift in $\lambda_{\mathrm{fmax}}$ of TNS and DNS in PSQA corresponds well to the observed marked increase in catalytic activity of PSQA for the decarboxylation of 6-nitrobenzisoxazole-3-carboxylate anion. The emission anisotropy, $r$, reflecting the immobility of TNS ionically bound to PSQA gradually increases with increasing $x$ in PSQA, while $r$ for DNS ionically and covalently bound to PSQA rapidly increases up to $x=8 \%$ and then levels off for a further increase in $x$. Almost free penetration of small molecules can be supposed only for PSQA with $2 \%$ divinylbenzene. Non-stationary-state measurements of emission anisotropy gave rotational relaxation times $\rho$ in the range of $50-130 \mathrm{~ns}$ for the probes in cross-linked polystyrene beads.

KEY WORDS Fluorescent Probe / 6-p-Toluidino-2-naphthalenesulfonate / 5-Dimethylamino-1-naphthalenesulfonate / Cross-Linked Polystyrene / Cationic Gel / Hydrophobicity / Depolarization / Emission Anisotropy / Rotational Relaxation / Polymer Catalyst /
\end{abstract}

Cross-linked polystyrene gels show considerable promise as support materials for various catalysts, photochemical sensitizers and organic reagents as well as beads for gel-permeation chromatography. The influence of the structure of polystyrene matrices on the reactivity and mobility of molecules bound to the matrices has been poorly investigated so far.

Recently, cross-linked polystyrene resins having quaternized ammonium chloride (PSQA) ${ }^{1}$ have been found to show much higher catalytic activities for the decarboxylation of 6-nitrobenzisoxazole-3carboxylate anion in comparison with a linearpolymeric catalyst or a cationic micelle with the same functional groups. The hydrophobic microenvironment due to cross-linked matrices was suggested to be a cause for this catalytic activity. ${ }^{1}$ We have used 6$p$-toluidino-2-naphthalenesulfonate (TNS) ${ }^{2-5}$ and 5-dimethylamino-1-naphthalenesulfonate $\quad(\mathrm{DNS})^{6}$ as fluorescence probes for evaluating microscopic hydrophobicity of the sites around the probes in these cationic cross-linked gels. In order to measure the mobility of functional groups bound to matrices, cross-linked polystyrene beads having covalently bound 5-dimethylamino-1-naphthalenesulfonamide groups (PSDNS) have also been prepared and the fluorescence depolarization technique has been applied to them.

The applicability of fluorescent-probe technique to the heterogeneous cross-linked gel systems will be discussed first in the present paper, and then the static and dynamic structure of cross-linked gels influencing their catalytic activity will be considered from the results of maximum wavelength $\left(\lambda_{\mathrm{fmax}}\right)$, intensity $\left(I_{\mathrm{f}}\right)$ and emission anisotropy $(r)$ of the 


\section{K. HORIE et al.}

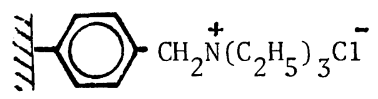

PSQA

fluorescences of the probes in cross-linked gels.

\section{FLUORESCENCE MEASUREMENTS FOR HETEROGENEOUS GEL SYSTEMS}

Cross-linked polystyrene beads with quaternary triethylammonium chloride groups (PSQA) were prepared with the same method as previously described. ${ }^{1}$ The content of divinylbenzene in the gel, percentage of ring substitution by quaternary ammonium group, and average particle diameter are indicated in Table $I$. The stability of colloidal dispersions of PSQA in water in the presence of $10^{-4}$ $M$ TNS was examined from the dependence of

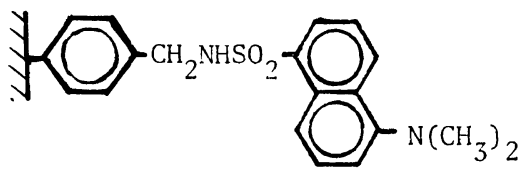

PSDNS

fluorescence intensity $I_{\mathrm{f}}$ relative to initial value $I_{\mathrm{f} 0}$ on the standing time of samples without stirring. Figure 1 shows that the sedimentation velocity depends on particle diameter and beads with the largest diameter (PS2QA, 30-70 $\mu \mathrm{m}$ ) were sedimented within a few minutes. The same figure shows that stable colloidal dispersions of PSQA in water for at least several minutes were obtained by grinding the beads in an agate mortar with a pestle up to average diameter less than $1 \mu \mathrm{m}$. The grinding of PSQA beads has enabled us to get fluorescent spectra of the gel-probe systems without any change in maximum wavelength $\lambda_{\mathrm{fmax}}$.

Typical absorption spectra for probes in cross-

Table I. Maximum wavelength $\lambda_{\text {fmax }}$, intensity $I_{\mathrm{f}}$, and quantum yield $\phi_{\mathrm{f}}$ for the fluorescence of TNS and DNS in water with cross-linked gels or some model compounds

\begin{tabular}{|c|c|c|c|c|c|c|c|}
\hline \multirow{4}{*}{ No. } & \multicolumn{2}{|c|}{ PSQA or model compounds } & \multirow{2}{*}{\multicolumn{2}{|c|}{$6,2-\mathrm{TNS}$}} & \multirow{2}{*}{\multicolumn{3}{|c|}{ 5,1-DNS }} \\
\hline & & \multirow{3}{*}{$\begin{array}{c}\begin{array}{c}\text { Original } \\
\text { particle } \\
\text { diameter }\end{array} \\
\mu \mathrm{m}\end{array}$} & & & & & \\
\hline & & & & \multirow{2}{*}{$I_{\mathrm{f}}\left(\phi_{\mathrm{f}}\right)$} & \multirow{2}{*}{$\frac{\lambda_{\mathrm{f} \max }}{\mathrm{nm}}$} & \multicolumn{2}{|c|}{$I_{\mathrm{f}}\left(\phi_{\mathrm{f}}\right)$} \\
\hline & & & $\mathrm{nm}$ & & & & \\
\hline 0 & Probe only & & 500 & $0.1(0.0009)$ & 510 & 11 & $(0.36)$ \\
\hline 1 & PS0QA70 & & 456 & $38 \quad(0.34)$ & 482 & 17 & $(0.56)$ \\
\hline 2 & PS2QA79(B) ${ }^{b}$ & $30-70$ & 448 & 42 & 480 & 16 & \\
\hline 3 & PS8QA92 $(\mathrm{H})^{\mathrm{c}}$ & $5-20$ & 443 & 8.1 & 467 & 5.1 & \\
\hline 4 & PS8QA88 $(T)^{d}$ & $5-20$ & 444 & 6.5 & 470 & 3.0 & \\
\hline 5 & PS15QA40 $(\mathrm{H})^{\mathrm{c}}$ & $1-2$ & 435 & 26 & 453 & 13 & \\
\hline 6 & PS15QA78 $(\mathrm{T})^{\mathrm{d}}$ & $1-2$ & 440 & 15 & 465 & 6.2 & \\
\hline 7 & PS15QA54 $(\mathrm{H})^{\mathrm{c}}$ & $5-20$ & 440 & 15 & 460 & 6.8 & \\
\hline 8 & PS15QA78 $(\mathrm{T})^{\mathrm{d}}$ & $5-20$ & 442 & 15 & 467 & 10 & \\
\hline 9 & PS28QA20 $(\mathrm{H})^{\mathrm{c}}$ & $5-20$ & 433 & 3.6 & 453 & 3.8 & \\
\hline 10 & PS28QA45 $(T)^{d}$ & $5-20$ & 433 & 5.5 & 455 & 2.2 & \\
\hline 11 & PS55QA13 $(\mathrm{H})^{\mathrm{c}}$ & $5-20$ & 437 & 1.9 & 470 & 1.3 & \\
\hline 12 & PS55QA23 $(\mathrm{T})^{\mathrm{d}}$ & $5-20$ & 437 & 2.2 & 465 & 1.1 & \\
\hline 13 & $\mathrm{BTACl}$ & & 490 & $0.15(0.0014)$ & 506 & 11 & $(0.36)$ \\
\hline 14 & CTABr & & 405 & $65 \quad(0.59)$ & 480 & 26 & $(0.85)$ \\
\hline
\end{tabular}

${ }^{\text {a }}$ Figure or double figures between PS and QA hereafter indicate $\%$ divinylbenzene in monomer composition, and double figures after QA indicate \% ring substitution by quaternary ammonium groups.

b Bio-Beads S-X2 were used for starting material.

c Hexane was used as diluent in the suspension polymerization process.

d Toluene was used as diluent in the suspension polymerization process. 


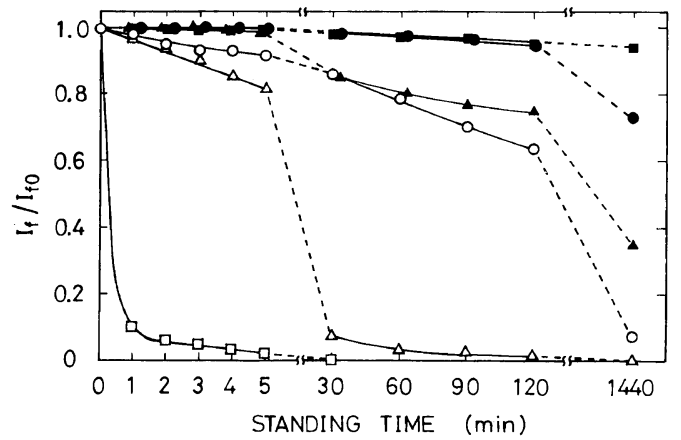

Figure 1. The change of $I_{\mathrm{f}} / I_{\mathrm{f} 0}$ for TNS in water with unground $(\bigcirc, \triangle, \square)$ and ground $(\boldsymbol{O}, \boldsymbol{\Delta}, \boldsymbol{\square})$ gel beads according to the standing time of samples without stirring. Gels: $\bigcirc$, PS15QA40 (No. 5); $\triangle, \boldsymbol{\Delta}$, PS15QA78 (No. 8); $\square, \boldsymbol{\square}$, PS2QA79 (No. 2).

linked gels are shown in Figure 2. The absorption maximum of TNS with soluble linear polystyrene having triethylammonium chloride PSOQA (Curve B) is shifted a little to a longer wavelength compared with a reference TNS solution in water (Curve A). As PSQA beads prior to grinding transmit no light, absorption spectra cannot be observed for the TNS ionically associated with unground PSQA, even when the beads are well dispersed in water. On the other hand, the ground PSQA beads do transmit light to a great extent, giving absorption spectra corresponding to TNS ionically associated with triethylammonium cation as shown for TNSPS15QA (Curve C) in Figure 2.

The time-courses of the variation in concentration

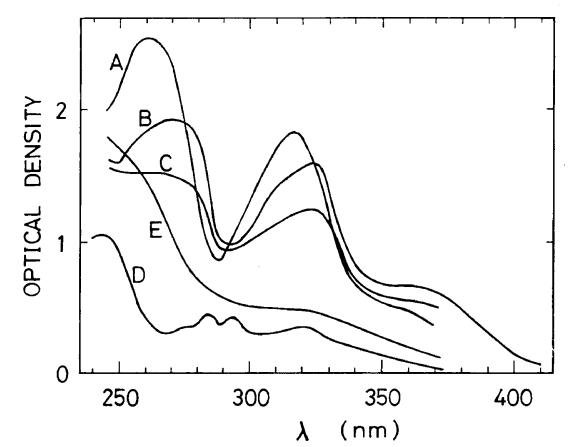

Figure 2. Absorption spectra of TNS in water without additive (A), with PS0QA (B), with ground PS15QA (No. 5) (C), and absorption spectra of DNS in water without additive (D) and with PSOQA (E). Concentrations of TNS and DNS, $1 \times 10^{-4} \mathrm{M}$. of free TNS in water were measured from absorption spectra for TNS with a stoichiometric amount of unground PSQA beads. The rate constant for ionic association $k$ and equilibrium constant for the association $K$ were estimated to be $k=1-10 \mathrm{M}^{-1}$ $\mathrm{s}^{-1}$ and $K=8 \times 10^{5} \mathrm{M}^{-1}$ for TNS-PS15QA system from the initial decrease in concentration of free TNS and from its final equilibrium concentration respectively. These values will assure the quantitative association of the probes with PSQA within an hour following the addition of probes for the experimental conditions described below.

Typical emission spectra for fluorescent probes in the cross-linked gels are shown in Figure 3. The position of fluorescence maxima shifts to a shorter wavelength with increasing divinylbenzene content $x$ in PSQA. The $\lambda_{\text {fmax }}$ for covalently bound DNS to cross-linked gels $\left(\lambda_{\text {f } \max }=490-510 \mathrm{~nm}\right.$ and 470 $490 \mathrm{~nm}$ for PSDNS in water and in tetrahydrofuran, respectively) also shows blue shifts in comparison with $\lambda_{\text {fmax }}$ for 5-dimethylamino-1-naphthalenesulfonamide (low-molecular-weight model) in water $(570 \mathrm{~nm}) .{ }^{6}$ Fluorescence spectrum for TNS in water is not shown in Figure 3 due to the very low fluorescence quantum yield $\left(\phi_{\mathrm{f}}=0.0009\right)$ which is one of the characteristics of TNS as a hydrophobic probe, while DNS in water gives a fluorescence spectrum with a sufficient quantum yield. Fluorescence quantum yields $\phi_{\mathrm{f}}$ for soluble systems were determined from measured fluorescence intensities $I_{\mathrm{f}}$ with the values of $\phi_{\mathrm{f}}=0.34$ for TNS in methanol ${ }^{3}$ and $\phi_{\mathrm{f}}=0.36$ for DNS in water. ${ }^{6}$

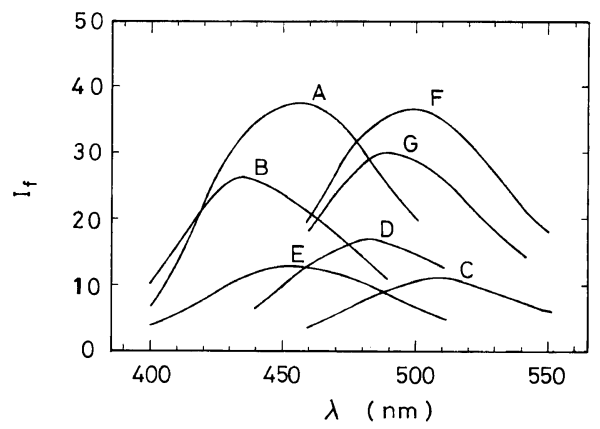

Figure 3. Fluorescence spectra of TNS in water with PS0QA (A), with PS15QA (No. 5) (B), of DNS in water without additive (C), with PS0QA (D), with PS15QA (No. 5) (E), and of PS15DNS (No. 5) in water (F) and in THF $(\mathrm{G})$. Concentrations of TNS and DNS, $1 \times 10^{-5} \mathrm{M}$; excitation wavelength, $320 \mathrm{~nm}$. 
Figure 4 shows the dependence of fluorescence intensity $I_{\mathrm{f}}$ on DNS concentration [DNS] at fixed PSQA concentrations. The proportionality between $I_{\mathrm{f}}$ and [DNS] holds for $[\mathrm{DNS}] \leqq 1 \times 10^{-5} \mathrm{M}$; for higher DNS concentration, however, the influence of concentration quenching is observed. As for the dependence of $I_{\mathrm{f}}$ on PSQA concentration at fixed probe concentrations, $I_{\mathrm{f}}$ increases gradually with an increase in the apparent concentration of ammonium cation [QA], and approaches constant values for $[\mathrm{QA}]$ ten times greater than [TNS] or [DNS]. The emission anisotropy, $r$, reflecting the rotational relaxation of DNS ionically associated with PSQA, gave constant values independent of DNS concentration for $[\mathrm{DNS}] \leqq 1 \times 10^{-5} \mathrm{M}$.

According to the above results, the fluorescence measurements hereafter were carried out at room temperature for ground PSQA beads with probe concentration of $1 \times 10^{-5} \mathrm{M}$, [QA] of $3 \times 10^{-4}$ $8 \times 10^{-4}$ eq $\mathrm{dm}^{-3}$, and excitation wavelength of $320 \mathrm{~nm}$.

\section{HYDROPHOBICITY OF BINDING SITES IN GELS}

The maximum wavelength $\lambda_{\mathrm{fmax}}$ and intensity $I_{\mathrm{f}}$ of fluorescences for TNS-PSQA and DNS-PSQA systems are summarized in Table $I$. The values for probes in aqueous solutions of benzyltriethylammonium chloride (BTACl) as a monomer model and of cetyltriethylammonium bromide (CTABr) as a micelle-forming cation are also given in Table I. For both probes, $\lambda_{\mathrm{fmax}}$ is shifted to a shorter wavelength, when the probe solution in water with no additive (No. 0) is changed to a solution with linear-

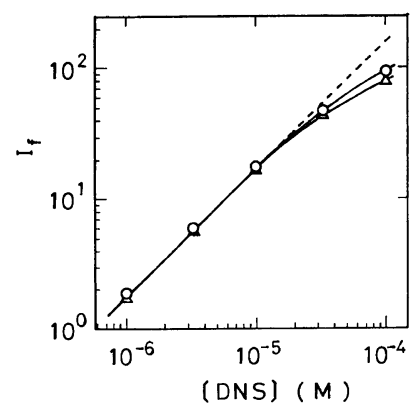

Figure 4. Relationship between $I_{\mathrm{f}}$ and [DNS] at fixed concentrations of PS0QA $(O)$ and PS2QA $(\triangle)$ in water. polystyrene cation (No. 1) and further to solutions with cross-linked gels PSQA in the order of increasing divinylbenzene content. As a decrease in solvent polarity is well known to induce blue shifts of $\lambda_{\text {fmax }}$ for TNS and DNS, these changes in $\lambda_{\text {fmax }}$ can be attributed to the increase in hydrophobicity of microenvironment around the probes with an increase in divinylbenzene content in the cross-linked gels. In case of PS55QA, only the fluorescence from probes located on the surface of the beads is supposed to be detectable. It is noteworthy that at a fixed divinylbenzene content, a shorter $\lambda_{\text {fmax }}$ was obtained for polystyrene gels prepared in suspension polymerization using hexane compared with $\lambda_{\text {fmax }}$ for those prepared with toluene. This may be related to the macroreticular structure ${ }^{7,8}$ supposed for the cross-linked beads polymerized in the presence of hexane which dissolves monomers but does not solvate the resulting network polymer. Further blue shifts of $\lambda_{\text {f } \max }$ in several nanometers were observed after 48 hours for TNS with PS8QA, PS15QA, PS28QA, and PS55QA, probably due to the transfer of TNS to deeper equilibrium sites in the gels.

The fact that $I_{\mathrm{f}}$ for PS2QA are comparable to $I_{\mathrm{f}}$ for soluble PS0QA suggests that excitation and emission lights are thoroughly transmitted by the colloidal suspension of the ground PS2QA. The polystyrene beads with higher divinylbenzene contents reflect light to some extent even after being ground, resulting in the decrease in $I_{\mathrm{f}}$. The values of $I_{\mathrm{f}}$ seem to depend complicatedly on surface area of the ground beads, depth of the surface which transmits light, formation of solvent channels, and the distribution of probes in the beads.

The results with $\mathrm{BTACl}$, which is a monomer model for PSQA, are similar to the case without additive, suggesting that the hydrophobicity observed for PSQA-probe systems is caused mainly by polystyrene chains around the probes. The addition of micelle-forming $\mathrm{CTABr}$ induces the largest blue shift of $\lambda_{\text {fmax }}$ for the case of TNS, while it does not induce a remarkable blue shift of $\lambda_{\text {fmax }}$ in the case of DNS. The interaction of $p$-tolyl group in TNS with arrayed hydrocarbon chains of CTABr may be important for the interaction of TNS with the micellar cation. This difference in the extent of the blue shift between TNS and DNS reminds us of the statement ${ }^{9}$ that a fluorescent probe does not always give absolute hydrophobicity of the surrounding site but the observed information depends on a mutual 
interaction of the probe and the site.

A plausible picture for the excited-state behavior of arylaminonaphthalenesulfonate (ANS) derivatives has been presented by Kosower et al. ${ }^{5}$ They have shown from the evaluation of emission data in connection with a solvent polarity parameter $E_{\mathrm{T}}(30)$ that there are two emitting states $S_{1, \mathrm{np}}$ and $S_{1, \mathrm{ct}}$ $(\mathrm{np}=$ nonplanar and $\mathrm{ct}=$ charge transfer) which respond differently to solvent polarity and viscosity. The empirical polarity parameter $E_{\mathrm{T}}(30)$ was proposed by Dimroth et al. ${ }^{10}$ as a value of energy corresponding to absorption maximum of betaine 30 in the solvent concerned. As is shown in Figure 1 of ref 5 , even in non-viscous solvents as well as in viscous solvents fluorescence of TNS with $\lambda_{\text {fmax }} \leqq 442$ $\mathrm{nm}\left(E_{\mathrm{F}} \geqq 64.5 \mathrm{kcal} \mathrm{mol}^{-1}\right)$ can be regarded as the emission from $S_{1, n p}$. For the fluorescence of TNS with $\lambda_{\text {f } \max }>442 \mathrm{~nm}$, there are two possibilities concerning the emitting state: $S_{1, \mathrm{np}}$ in the viscous solvent or $\mathrm{S}_{1, \mathrm{ct}}$ in the non-viscous solvent. As the emission from $S_{1, c t}$ is accompanied by a marked decrease in $\phi_{\mathrm{f}}$, the large $I_{\mathrm{f}}$ values observed in the present cases with PSQA (especially No. 1 and 2) support the identification of the emitting state as $\mathrm{S}_{1, \mathrm{np}}$ for these cases. Thus, the reduced mobility of TNS associated with polymer matrices restricts the electron-transfer process from $S_{1, \mathrm{np}}$ to $S_{1, \mathrm{ct}}$ within lifetime of the excited state, and the hydrophobicity of microenvironment around the probe will be estimated by using the linear relationship between $E_{\mathrm{T}}(30)$ and reciprocal $\lambda_{\text {f } \max }$ for $\mathrm{S}_{1, \mathrm{np}}$ given in Figure 1 of ref 5. In the case of DNS, the emitting state is the same in any solvent because of the absence of $\mathrm{N}$-aryl group. The hydrophobicity around DNS in PSQA

Table II. Hydrophobicity around the probes in crosslinked gels in water

\begin{tabular}{lcc}
\hline \multirow{2}{*}{ PSQA } & \multicolumn{2}{c}{$E_{\mathrm{T}}(30) / \mathrm{kcal} \mathrm{mol}^{-1}$} \\
\cline { 2 - 3 } & $\begin{array}{c}\text { From } \lambda_{\text {fmax }} \\
\text { of TNS }\end{array}$ & $\begin{array}{c}\text { From } \lambda_{\text {fmax }} \\
\text { of DNS }\end{array}$ \\
\hline PS0QA70 & 55 & 52 \\
PS2QA79 (B) & 53 & 52 \\
PS8QA92 (H) & 51 & 47 \\
PS15QA40 (H) & 47 & 43 \\
PS28QA20 (H) & 46 & 43 \\
PS55QA13 (H) & 48 & 47 \\
\hline
\end{tabular}

will also be estimated from the relationship between $E_{\mathrm{T}}(30)$ and reciprocal $\lambda_{\text {fmax }}$ of DNS. ${ }^{6}$

The hydrophobicities around the probes in PSQA in water are listed in Table II in terms of $E_{\mathrm{T}}(30)$. The lowest $E_{\mathrm{T}}(30)$ values for PS15QA(H) and PS28QA (H) are compared with that for binding site of apomyoglobin ( $42 \mathrm{kcal} \mathrm{mol}^{-1}$ from $\lambda_{\text {fmax }}$ of TNS). ${ }^{9}$

As already mentioned, the cationic-polystyrene beads PSQA exhibit remarkable catalytic activities for the decarboxylation of 6-nitrobenzisoxazole-3carboxylate anion (S) in water. A comparison of $\lambda_{\text {fmax }}$ with the rate constant $k_{2}$ in eq $1^{1}$ for the catalytic decarboxylation of the substrate (S) by PSQA (C) is shown in Figure 5.

$$
\begin{aligned}
& \mathrm{S}+\mathrm{C} \underset{k_{-1}}{\stackrel{k_{1}}{\rightleftharpoons}} \mathrm{SC} \stackrel{k_{2}}{\longrightarrow} \mathrm{PC} \underset{k_{-3}}{\stackrel{k_{3},+\mathrm{S}}{\rightleftarrows}} \\
& \mathrm{SC}+\text { Product }(\mathrm{P})
\end{aligned}
$$

In cases of PSQA with divinylbenzene contents below $15 \%$, excellent correspondence between $k_{2}$ and $\lambda_{\text {fmax }}$ has been attained, showing that $k_{2}$ is controlled by the hydrophobicity of microenvironment around cationic sites in the cross-linked gels. The deviations observed for PS28QA and PS55QA might be caused by a decrease in the effective number of catalytic sites, since the diffusion of the substrate inside the

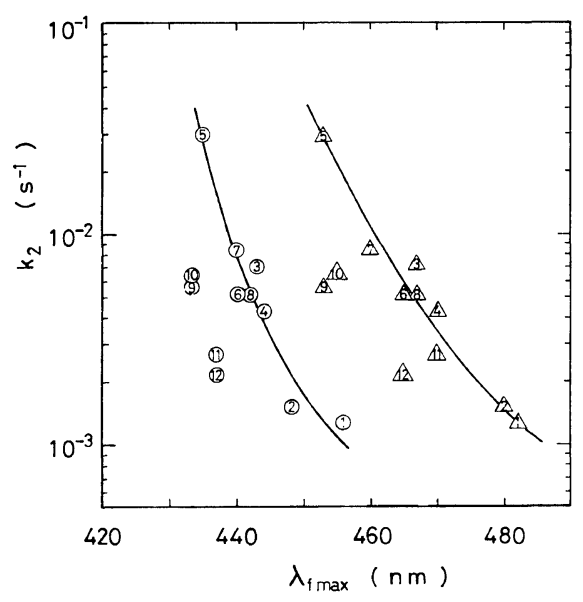

Figure 5. Relationship between the catalytic rate constant $k_{2}$ of PSQA and $\lambda_{\mathrm{f} \max }$ of TNS $(O)$ or DNS $(\triangle)$ in water in the presence of corresponding PSQA. Numbers for PSQA in circles and triangles are the same as in Table I. 
cross-linked gels is greatly restricted as a result of very high cross-linking density in these cases. This supposition is supported by the results of fluorescence depolarization of PSDNS as is discussed in the following section. The grinding of PS28QA20 $(\mathrm{H})$ having an original particle diameter of 5-20 $\mu \mathrm{m}$ to about $1 \mu \mathrm{m}$ increased its rate constant $k_{2}$ by about $50 \%$, but this is still much smaller than $3 \times 10^{-2} \mathrm{~s}^{-1}$ for the unground PS15QA40 $(\mathrm{H})$ with a particle diameter of $1-2 \mu \mathrm{m}$.

\section{MOBILITY OF PROBES IN GELS}

The rotational relaxation time $\rho$ of a fluorescent molecule attached to linear polymer chains has been obtained from measurements of fluorescence depolarization, ${ }^{11}$ but so far, there have been few applications of fluorescence-depolarization technique to cross-linked gel systems. ${ }^{12}$

The emission polarization $p$ or emission anisotropy $r$ can be calculated from eq 2 or $3,{ }^{13.14}$

$$
\begin{gathered}
p=\frac{I_{\|}-I_{\perp}}{I_{\|}+I_{\perp}}=\frac{I_{\mathrm{vv}}-G I_{\mathrm{vh}}}{I_{\mathrm{vv}}+G I_{\mathrm{vh}}} \\
r=\frac{I_{\|}-I_{\perp}}{I_{\|}+2 I_{\perp}}=\frac{I_{\|}-I_{\perp}}{I}=\frac{I_{\mathrm{vv}}-G I_{\mathrm{vh}}}{I_{\mathrm{vv}}+2 G I_{\mathrm{vh}}}
\end{gathered}
$$

where the intensities of emitted light polarized parallel $\left(I_{\|}\right)$and perpendicular $\left(I_{\perp}\right)$ to the exciting light are related to the observed intensities $I_{\mathrm{vv}}, I_{\mathrm{vh}}$, and a correction factor $G=I_{\mathrm{hv}} / I_{\mathrm{hh}}$ (subscripts $\mathrm{v}$ and $\mathrm{h}$ denote vertically and horizontally polarized lights, the first letter in the subscript refers to exciting light and the second refers to emitting light). Since the factor $G$ depended on fluorescence wavelength, it was determined for every $\lambda_{\mathrm{f}}$ by using a standard quinine sulfate solution. Figure 6 shows that the emission anisotropy $r$ for the probes in cross-linked gels depends on emission wavelength $\lambda_{\mathrm{f}}$ and increases with decreasing $\lambda_{\mathrm{f}}$, reflecting the inhomogeneity of cationic sites in the gels. The slope in $r-\lambda_{\mathrm{f}}$ plots may be used as a measure of existence of various states (inhomogeneity) of cationic sites in the gels.

In order to compare the mobility of probes in the gels with each other, the value of emission anisotropy at the wavelength of maximum fluorescence intensity was adopted as $r$ for each gel-probe system. The values of $r$ for TNS and DNS ionically associated with PSQA and for DNS covalently bound to crosslinked polystyrene (PSDNS) are summarized in

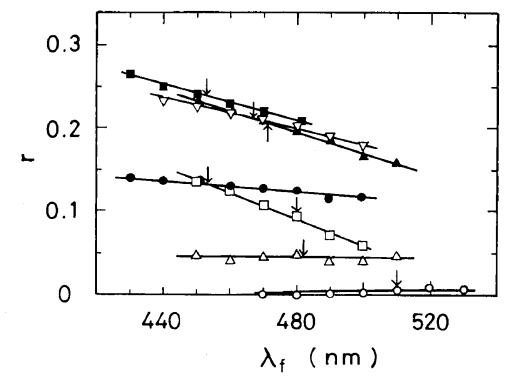

Figure 6. Wavelength dependence of emission anisotropy $r$ of DNS in water without additive $(O)$, with PS0QA $(\triangle)$, PS2QA $(\square)$, PS8QA (No. 3) $(\triangle)$ ), PS15QA

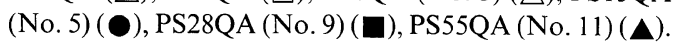
Arrow indicates the wavelength of maximum fluorescence intensity.

Table III, and are illustrated in Figures 7 and 8 against divinylbenzene content $x$ in the cross-linked gels.

In the case of TNS ionically associated with PSQA, $r$ for linear PS0QA is already larger than 0.1 and increases with increasing $x$ of PSQA. On the other hand, in those cases in which DNS is both ionically and covalently bound to the gels, $r$ is very small for linear PSOQA, and increases markedly with an increase in $x$ up to $8 \%$, and then it levels off or gradually decreases with a further increase in $x$. This difference would suggest a tight association of TNS due to its stronger hydrophobic interaction with polystyrene chains in comparison with the loose association of DNS. The curves for DNS in Figures 7 and 8 suggest that DNS molecule or dansylchloride used in the preparation of PSDNS, penetrates the cross-linked gels of $x<8 \%$ almost freely, but when $x$ becomes larger than $8 \%$ its penetration inside the cross-links seems to become difficult, and $r$ for this case reflects the mobility of detectable DNS attached to the surface and solvent channels of the gels. The fact that the values of $r$ for PSDNS in tetrahydrofuran (THF) are smaller than those in water may be explained by the swellability of the polymer in THF. There seems to be no difference in mobilities of the probes in gels prepared with different diluents during the polymerization process, except for the case of PS15QA40 (H) which exhibits a remarkable deviation of $r$ in the direction of a higher mobility of DNS. Observations of PS15QA40 (H) beads by a scanning electron microscope (SEM) and by a transmission electron microscope (TEM) in Figure 9 
Table III. Emission anisotropy $r$ for ionically and covalently bound probes in cross-linked gels or in some model compounds

\begin{tabular}{|c|c|c|c|c|c|}
\hline \multirow{2}{*}{ No. } & \multirow{2}{*}{$\begin{array}{l}\text { PSQA, PSDNS or } \\
\text { model compounds }\end{array}$} & \multicolumn{4}{|c|}{$r$} \\
\hline & & $\begin{array}{l}\text { TNS-PSQA } \\
\text { in water }\end{array}$ & $\begin{array}{l}\text { DNS-PSQA } \\
\text { in water }\end{array}$ & $\begin{array}{l}\text { PSDNS } \\
\text { in water }\end{array}$ & $\begin{array}{l}\text { PSDNS } \\
\text { in THF }\end{array}$ \\
\hline 0 & Probe only & - & 0.003 & - & - \\
\hline 1 & PS0 & 0.12 & 0.046 & & \\
\hline 2 & PS2 (B) & 0.15 & 0.094 & 0.071 & 0.039 \\
\hline 3 & PS8 (H) & 0.18 & 0.21 & 0.25 & 0.18 \\
\hline 4 & PS8 (T) & 0.20 & 0.23 & 0.22 & 0.17 \\
\hline 5 & PS15 (H) & 0.16 & 0.13 & 0.13 & 0.12 \\
\hline 6 & PS15(T) & 0.17 & 0.21 & 0.23 & 0.19 \\
\hline 7 & PS15 (H) & 0.17 & 0.19 & 0.24 & 0.17 \\
\hline 8 & PS15 (T) & 0.20 & 0.20 & & \\
\hline 9 & PS28 (H) & 0.24 & 0.23 & 0.23 & 0.14 \\
\hline 10 & PS28 (T) & 0.24 & 0.22 & 0.24 & 0.16 \\
\hline 11 & PS55 (H) & 0.27 & 0.21 & 0.20 & 0.13 \\
\hline 12 & PS55 (T) & 0.30 & 0.23 & 0.19 & 0.086 \\
\hline 13 & $\mathrm{BTACl}$ & - & 0.009 & - & - \\
\hline 14 & $\mathrm{CTABr}$ & 0.14 & 0.012 & - & - \\
\hline
\end{tabular}

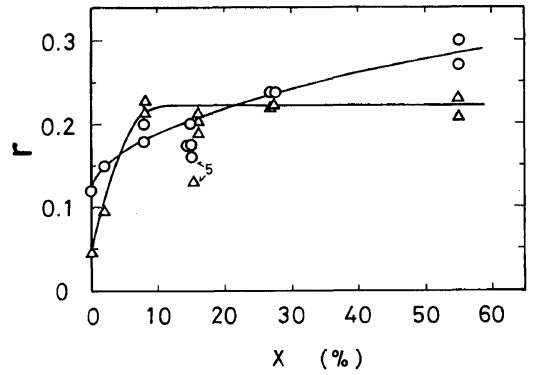

Figure 7. Dependence of emission anisotropy $r$ of TNS $(\bigcirc)$ or DNS $(\triangle)$ ionically associated with PSQA on the divinylbenzene content $x$ of PSQA.

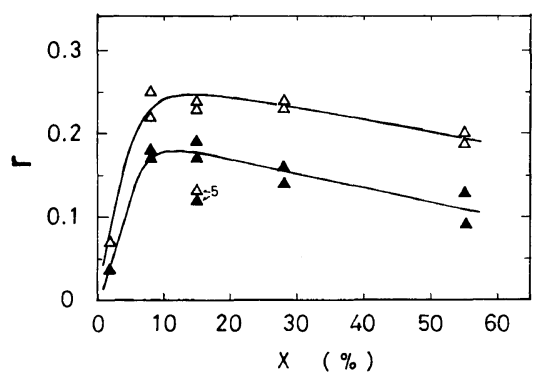

Figure 8. Dependence of emission anisotropy $r$ of DNS group of PSDNS in water $(\triangle)$ or in $\operatorname{THF}(\boldsymbol{\Delta})$ on the divinylbenzene content $x$ of PSDNS. revealed macroporous structure of the beads with numerous thick solvent channels inside the gel, compared with the structure for beads with the same divinylbenzene content and particle diameter but prepared with toluene (PS15QA78 (T)) in Figure 10.

In order to estimate the rotational relaxation times $\rho$ of fluorescent probes in some cross-linked gels, measurement of time-dependent polarized fluorescence intensities $I_{\|}(t)$ and $I_{\perp}(t)$ at $25^{\circ} \mathrm{C}$ was carried out with an ORTEC Single Photon Counting System at the Mitsubishi-Kasei Institute of Life Sciences. The excitation wavelength was $360 \mathrm{~nm}$, with a halfpulse-width of $3 \mathrm{~ns}$. Fluorescence lifetime $\tau$, intrinsic emission anisotropy $r_{0}$, and dynamic rotational relaxation time $\rho_{\text {dyn }}$ were then calculated with an online type minicomputer according to eq 4 and 5 .

$$
\begin{aligned}
& I(t)=I_{\|}(t)+2 I_{\perp}(t)=I_{0} \exp (-t / \tau) \\
& r(t)=\left\{I_{\|}(t)-I_{\perp}(t)\right\} / I(t)=r_{0} \exp \left(-3 t / \rho_{\mathrm{dyn}}\right)
\end{aligned}
$$

These values are listed in Table IV. The stationarystate rotational relaxation time $\rho_{\text {stat }}$ calculated according to eq 6 using $r$ from stationary-state meas-

$$
1 / r=\left(1 / r_{0}\right)\left(1+3 \tau / \rho_{\text {stat }}\right)
$$

urements together with the above $\tau$ and $r_{0}$ is also given in Table IV.

Values of fluorescence lifetimes $\tau$ of probes in 


\section{K. HORIE et al.}
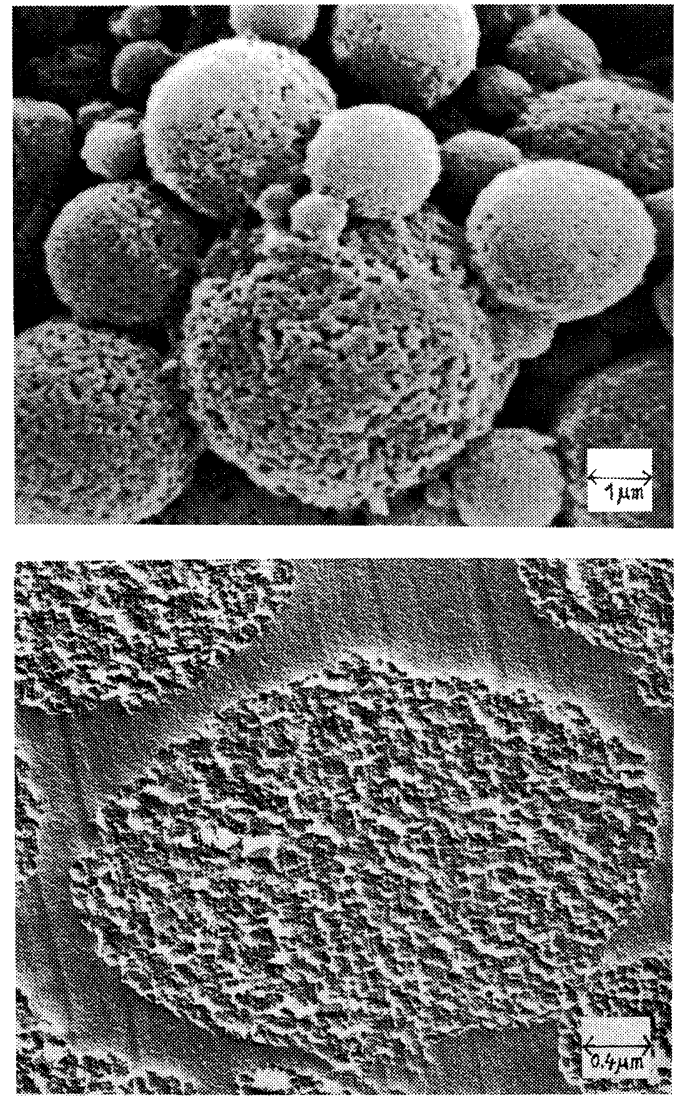

Figure 9. PS15QA40 $(\mathrm{H})$ beads observed by SEM after gold coating (upper photo, $\times 16,000$ ) and by TEM after platinum-palladium shadowing (lower photo, $\times 40,000$ ).
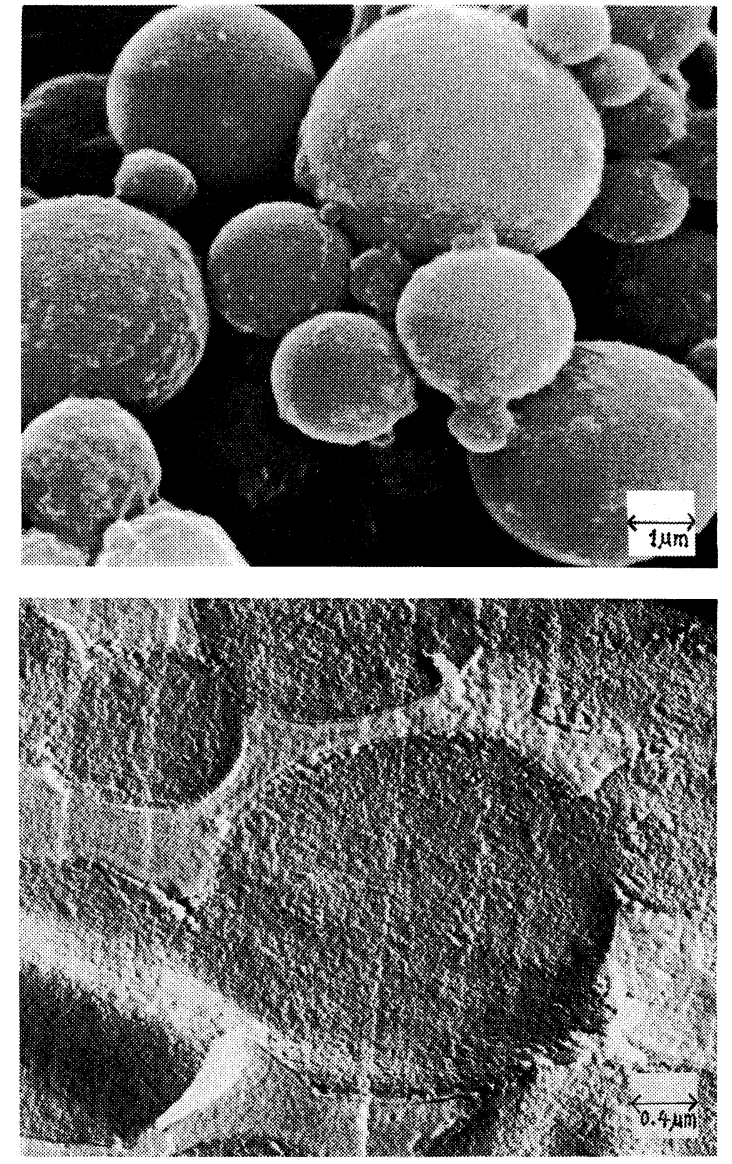

Figure 10. PS15QA78 (T) (No. 6) beads observed by SEM after gold coating (upper photo, $\times 16,000$ ) and by TEM after platinum-palladium shadowing (lower photo, $\times 40,000)$.

Table IV. Rotational relaxation time $\rho$ for fluorescent probes in cross-linked gels

\begin{tabular}{|c|c|c|c|c|c|c|c|}
\hline \multirow{3}{*}{ System } & \multicolumn{4}{|c|}{ Dynamic measurements } & \multicolumn{3}{|c|}{ Stationary-state measurements } \\
\hline & $\lambda_{\mathrm{f}}$ & $\tau$ & & $\rho_{\mathrm{dyn}}$ & $\lambda_{\mathrm{f} \max }$ & & $\rho_{\text {stat }}$ \\
\hline & $\mathrm{nm}$ & ns & & ns & $\mathrm{nm}$ & & ns \\
\hline TNS-PS2QA79 (B) & 460 & 7.3 & 0.177 & 81 & 448 & 0.147 & 107 \\
\hline DNS-PS0QA70 & 460 & 20.0 & 0.159 & 53 & 482 & 0.046 & 25 \\
\hline DNS-PS2QA79 (B) & 460 & 17.1 & 0.137 & 131 & 480 & 0.094 & 112 \\
\hline DNS-PS15QA40 (H) & 460 & 13.2 & 0.176 & 78 & 453 & 0.134 & 126 \\
\hline PS15DNS (No. 5) in water & 480 & 3.5 & 0.154 & 93 & 500 & 0.128 & 52 \\
\hline PS15DNS (No. 5) in THF & 480 & 8.2 & 0.172 & 90 & 490 & 0.120 & 57 \\
\hline
\end{tabular}

cross-linked gels agree rather well with those reported for probes in water without additives (TNS,
$10 \mathrm{~ns}$; DNS, $16.5 \mathrm{~ns}$; DNSulfonamide, $3.3 \mathrm{~ns}) .{ }^{5.6}$ The intrinsic emission anisotropies $r_{0}$ obtained for these 
systems are very small compared with 0.4 supposed for vertically polarized excitation of the usual dyes in solution. Results similar to ours have been observed for intrinsic polarization for solutions of linear polymers with some fluorescent probes..$^{13}$ The values of rotational relaxation times $\rho$ of probes in the cross-linked gels in Table IV lie between 50-130 ns. These values are about 1.5-4 times larger than rotational correlation times for the nitroxide spin probe covalently bound to non-swelled cross-linked polystyrenes, ${ }^{15}$ and are about $30-300$ times larger than the values for spin probes covalently bound to well-swelled cross-linked polystyrene. ${ }^{16}$ This difference may be due to the stronger hydrophobic interaction of TNS and DNS with polystyrene chains and their larger hydrodynamic volumes compared with the nitroxide probe. The values of $\rho$ in Table IV are about ten times larger than $\rho$ for fluorescent probes covalently attached to linear polymers diluted in good solvents, ${ }^{13}$ but are in the same order of magnitude with $\rho$ for fluorescent probes in concentrated polyacrylamide solution. ${ }^{17}$ No significant influence of the nature of binding (ionic or covalent) on $\rho$ was observed in Table IV.

The agreement of $\rho_{\text {dyn }}$ with corresponding $\rho_{\text {stat }}$ is not always good as far as observed in Table IV, probably due to the inhomogeneity of binding sites in cross-linked gels and the differences in the monitored emission wavelength $\lambda_{\mathrm{f}}$ for dynamic and stationary-state measurements. Though the present results for $\rho$ are quite insufficient for a detailed discussion on the effect of structure of gels on $\rho$, it can be said that the overall characteristics of the influence of cross-linking structure on the mobility of binding sites can be visualized by the stationary-state measurements of emission anisotropy $r$ as shown in Figures 7 and 8.

In conclusion, fluorescent probes such as TNS and DNS are found to be effective for characterizing the hydrophobicity and some dynamic features of crosslinked polymers. The cross-linked polystyrene beads PS15QA40 $(\mathrm{H})$ showing the largest catalytic rate constant $k_{2}$ for decarboxylation should have a macroporous structure with numerous thick solvent channels inside the gel anticipated for macroreticular resins. This structure may assure the coexistence of high hydrophobicity (Figure 5), relatively high mobility (Figures 7 and 8), and homogeneity (Figure 6) of binding sites in the gel.

\section{EXPERIMENTAL}

\section{Materials}

Cross-Linked Polystyrenes with Quaternary Ammonium Groups (PSQA). The PSQA beads were prepared by the same method previously described. ${ }^{1}$ Hexane (non-solvent for polymer) or toluene (solvent for polymer) was used as a diluent for the suspension polymerization of styrene with divinylbenzene. The particle diameter of the beads was controlled by changing the amount of poly(vinyl alcohol) as a protective colloid and the speed of agitation. Chloromethylation and subsequent ring substitution by quaternary triethylammonium group for the polystyrene beads were carried out in the same manner as in previous experiments.

Cross-Linked Polystyrenes with Dansyl Groups (PSDNS). The same chloromethylated polystyrene beads used for the preparation of PSQA were heated with potassium phthalimide in dimethylformamide at $50^{\circ} \mathrm{C}$ for 18 hours, then treated with $5 \%$ of hydrazine hydrate at $75^{\circ} \mathrm{C}$ for 24 hours. ${ }^{18}$ The resulting aminomethylated polystyrene beads (30$60 \%$ of ring substitution) reacted with 5dimethylamino-1-naphthalenesulfonyl chloride in tetrahydrofuran at room temperature overnight under stirring, showing the increasing fluorescence due to 5-dimethylamino-1-naphthalenesulfonamide groups attached to the cross-linked polystyrene beads. The percentages of ring substitution by DNS group were $2-15 \%$ according to elemental analysis.

\section{Probes}

The 6-p-toluidino-2-naphthalenesulfonic acid and 5-dimethylamino-1-naphthalenesulfonic acid were purchased from Eastman Kodak Co. and Tokyo Kasei Co., respectively, and used as received.

\section{Fluorescence Measurements}

A JASCO FP-550 type spectrofluorometer was used for measuring the $\lambda_{\mathrm{fmax}}$ and $I_{\mathrm{f}}$ as well as fluorescence depolarization in stationary states by the aid of polarizing filters. Corrections for fluorescence intensity and $G$ factor in depolarization measurements were carried out with $1 \times 10^{-5} \mathrm{M}$ quinine sulfate solution in water with $0.5 \mathrm{M}$ of sulfuric acid. The experimental conditions including the concentrations of probes and cross-linked gels are already given in the foregoing section. Since the 
fluorescence characteristics of these probes were not altered by any change in $\mathrm{pH}$ of the solution within the range of 5-9, measurements were carried out with systems using no buffer. The experimental details with an ORTEC Single Photon Counting System used for the non-stationary-state measurements of $\tau, r_{0}$, and $\rho_{\mathrm{dyn}}$ will be given elsewhere.

Acknowledgment. The authors are grateful to Dr. N. Wakayama of Mitsubishi-Kasei Institute of Life Sciences for measurements of $\tau, r_{0}$, and $\rho_{\mathrm{dyn}}$ and his helpful discussion. The authors are also indebted to Dr. M. Fukuda of Life Science Research Laboratory of Toyo Soda Kogyo Co. for photographing PSQA beads with SEM and TEM.

\section{REFERENCES}

1. N. Yamazaki, S. Nakahama, A. Hirao, and J. Kawabata, Polym. J., 12, 231 (1980).

2. L. Stryer, J. Mol. Biol., 13, 482 (1965).

3. W. O. McClure and G. M. Edelman, Biochemistry, 5, 1908 (1966).

4. D. C. Turner and L. Brand, Biochemistry, 7, 3381
(1968).

5. E. M. Kosower, H. Dodiuk, K. Tanizawa, M. Ottolenghi, and N. Orbach, J. Am. Chem. Soc.,97, 2167 (1975).

6. Y. H. Li, L. M. Chan, L. Tyer, R. T. Moody, C. M. Himel, and D. M. Hercules, J. Am. Chem. Soc., 97, 3118 (1975).

7. J. R. Millar, D. G. Smith, and T. R. E. Kressman, J. Chem. Soc., 304 (1965).

8. K. A. Kun and R. Kunin, J. Polym. Sci. A-1, 6, 2689 (1968).

9. H. Dodiuk, H. Kanety, and E. M. Kosower, J. Phys. Chem., 83, 515 (1979).

10. K. Dimroth and Ch. Reichardt, Justus Liebigs Ann. Chem., 663, 1 (1963).

11. H. Morawetz, Science, 203, 405 (1979).

12. D. J. Wedlock, G. O. Phillips, and J. K. Thomas, Polym. J., 11, 681 (1979).

13. A. M. North and I. Soutar, J. Chem. Soc. Faraday Trans. 1, 68, 1101 (1972).

14. E. D. Cehelnik, K. D. Mielenz, and R. A. Velapoldi, $J$. Res. Natl. Bur. Stand. Part A, 79, 1 (1975).

15. S. L. Regen, J. Am. Chem. Soc., 96, 5275 (1974).

16. S. L. Regen, Macromolecules, 8, 689 (1975).

17. A. Teramoto, S. Hiratsuka, and Y. Nishijima, $J$. Polym. Sci., A-2, 5, 37 (1967).

18. J. T. Sparrow, J. Org. Chem., 41, 1350 (1976). 